\title{
Software
}

\section{Sistemas integrados para bibliotecas públicas. Las propuestas de Innovative}

\author{
Por Ricardo Eíto-Brun
}

\begin{abstract}
Resumen: Se presentan los últimos desarrollos de la empresa Innovative Interfaces Inc., III, fabricante del sistema integrado Innopac Millenium para bibliotecas públicas. Las principales novedades están relacionadas con el acceso al opac y a los servicios de la biblioteca a través de dispositivos móviles, y herramientas para potenciar y facilitar la participación de los usuarios a través del opac y agilizar la prestación de servicios y la comunicación entre el personal de la biblioteca y la comunidad de usuarios.

Palabras clave: Innovación, Bibliotecas públicas, Autopréstamo, Opac, Innovative Interfaces Inc., Innopac Millenium.

Title: The evolution of integrated systems for public libraries. New developments from Innovative

Abstract: Innovative Interfaces Inc., III, maker of the Innopac Millen-

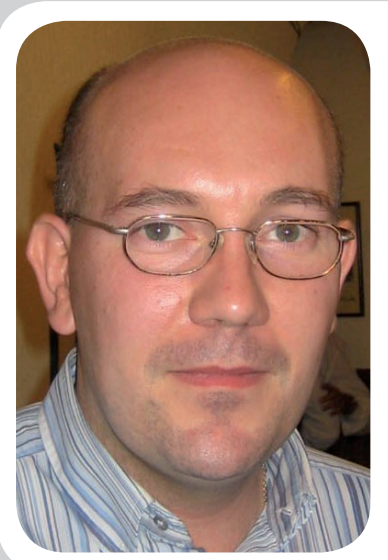

Ricardo É́to-Brun (Huesca, 1973), es profesor asociado en la Universidad Carlos III de Madrid y desarrolla su actividad profesional en el área de la calidad y gestión documental en el sector de las TIC. Es autor de dos libros sobre $\mathrm{xml}$ y de varios artículos sobre lenguajes de marcas, gestión documental y de contenidos.

nium integrated system for public libraries, has introduced new products that are primarily related to user access to opac and to library services via handheld devices (cellphone, PDA, etc.). There are also new tools to strengthen and facilitate user participation through opac and to provide more flexibility in service delivery and communication between the user community and library personnel.
\end{abstract}

Keywords: Innovation, Public libraries, Self check-out, Opac, Innovative Interfaces Inc., Innopac Millennium.

Eíto-Brun, Ricardo. "La evolución de los sistemas integrados para bibliotecas públicas. Las propuestas de Innovative". En: El profesional de la información, 2008, marzo-abril, v. 17, n. 2, pp. 225-230.

DOI: 10.3145/epi.2008.mar.14

EN LOS ÚLTIMOS AÑOS SE HAN PRODUCIDO AVANCES SIGNIFICATIVOS en los sistemas integrados de gestión bibliotecaria (en adelante SIGB), afectando especialmente al área de bibliotecas académicas. Entre ellos podemos destacar: la inclusión de módulos complementarios para la gestión de recursos-e (bases de datos y revistas electrónicas); la incorporación de software para la organización de repositorios institucionales, y los sistemas de búsqueda federada o metabuscadores.

En la mayoría de los casos se trata de aplicaciones complementarias a las funciones habituales que presentan, auque suelen permitir un uso independiente. En el área de las bibliotecas públicas, también se han incorporado mejoras significativas. La redacción de EPI tuvo ocasión de conocer las propuestas que la empresa Innovative Interfaces Inc., -fabricante del sistema Millenniumha diseñado para este área.

Estas iniciativas giran en torno a dos cuestiones que Innovative considera claves en los escenarios actuales: por una parte la necesidad de facilitar la interacción entre la biblioteca y sus usuarios, aplicando tecnologías relacionadas con la movilidad y la distribución de información a distintos tipos de dispositivos móviles (teléfonos, ordenadores de bolsillo o PDAs, etc). Por otra, la posibilidad de facilitar a

\begin{abstract}
"En el área de las bibliotecas públicas, las principales novedades técnicas buscan facilitar la interacción con y entre los usuarios de la biblioteca"
\end{abstract}

la comunidad de usuarios una mayor participación en las actividades de la biblioteca, dándoles un mayor protagonismo y haciendo posible una comunicación fluida entre los propios usuarios.

En los siguientes apartados resumimos brevemente algunos de los módulos complementarios Innopac Millennium, que muestran el 


\section{Ejemplo de plantilla para mensaje sms}

$<$ nombre de la biblioteca $>$. Tiene $<\mathrm{n}>$ ejemplares cuyo préstamo vencerá en las próximas 48 horas. Visite <el enlace del sitio web> para renovar o llame <número de teléfono $>$.

compromiso con la innovación característico de esta empresa.

\section{Integración con dispositivos móviles}

Millennium ha incorporado funciones para el envío de mensajes sms (short message service) a los usuarios. Se pueden enviar automáticamente en respuesta a determinados eventos como el vencimiento de préstamos, reclamaciones, avisos de recogida, disponibilidad de reservas, etc. El teléfono móvil del usuario se guarda como parte de su perfil, y el personal de la biblioteca puede configurar distintas alertas que se ejecutarán de forma desatendida. Los bibliotecarios también podrán enviarlos en cualquier otro momento pues no es un acto asociado forzosamente a un evento.

\section{"Los mensajes sms se pueden enviar automáticamente en respuesta a determinados situaciones: vencimiento de préstamos, reclamaciones, disponibilidad de reservas, etc."}

Los mensajes pueden codificarse con una extensión de hasta ciento sesenta caracteres, incluir urls y utilizar distintos juegos de caracteres. El sistema puede informar al personal de la biblioteca de aquellos que no se hayan podido entregar.

A parte del envío de mensajes y alertas mediante sms, Innovati- $v e$ ha desarrollado una interfaz de consulta para dispositivos móviles: teléfonos y ordenadores de bolsillo (PocketPC, Palm, etc.). Se trata del módulo AirPAC, que está disponible en el portfolio de productos desde hace varios años.

Los usuarios de AirPAC pueden acceder a sus datos, consultar información de vencimientos, sanciones, crear y cancelar reservas, así como renovar préstamos. Al igual que sucede con la interfaz de consulta web de Millennium, puede usarse con distintos idiomas. Podemos comprobar su funcionamiento en una biblioteca real conectándonos a través de un móvil o PDA a la url:

http://catalog.westervillelibrary. org/airpac/jsp/airpacIndex.jsp

\section{Circa: \\ control del inventario}

Es otro de los módulos desarrollados recientemente que resulta especialmente interesante para bibliotecas públicas. Es una aplicación portátil dirigida a los bibliotecarios para el control del inventario y se ejecuta en ordenadores de bolsillo. El personal de la biblioteca dispone de una herramienta donde puede registrar información sobre el estado y la ubicación de los fondos en los depósitos y en las salas de libre acceso. La información se puede volcar en tiempo real -o en un momento posterior- a la base de datos del catálogo Millennium.
Una posible aplicación es registrar los ejemplares que han sido utilizados por los usuarios en salas de acceso libre (normalmente, tras usarlos se depositan en ubicaciones reservadas para que posteriormente los bibliotecarios los coloquen en los estantes correctos). Estos datos, convenientemente registrados en Circa, resultan útiles para saber el uso que se está haciendo de los libros disponibles en libre acceso; información que normalmente no se registra y acaba perdiéndose.

\section{Self-registration y ExpressLane: autoservicio para los usuarios}

Self-registration consiste en un conjunto de formularios web donde los futuros usuarios pueden completar su registro como usuarios de la biblioteca. Los formularios incluirán un conjunto de campos y datos que se deben completar; tras rellenarlos se enviará una notificación a la biblioteca para que los valide. El módulo permite a la biblioteca personalizar los aspectos visuales y los campos que se muestran en estos formularios. Cuando el usuario completa su registro, el sistema le enviará un correo-e de confirmación.

Respecto a ExpressLane, se trata de un sistema para el préstamo desatendido. De esta forma se instalan unos terminales donde los usuarios que no tengan sanciones pueden completar operaciones de préstamo. Para ello el programa incorpora la capacidad de leer el carné del lector y el código de barras de los ejemplares, y registrar la operación de préstamo sin la intervención de un bibliotecario. Los usuarios también podrán ver a través de estos

\footnotetext{
Innovative usa la tecnología xml en Millennium AirPAC para distribuir datos del catálogo en respuesta a solicitudes recibidas desde distintos dispositivos de acceso inalámbricos (wireless). Los datos xml se convierten en páginas $\mathrm{html}$ formateadas para mostrarse en dispositivos tipo Palm, PocketPC o teléfonos móviles.
} 


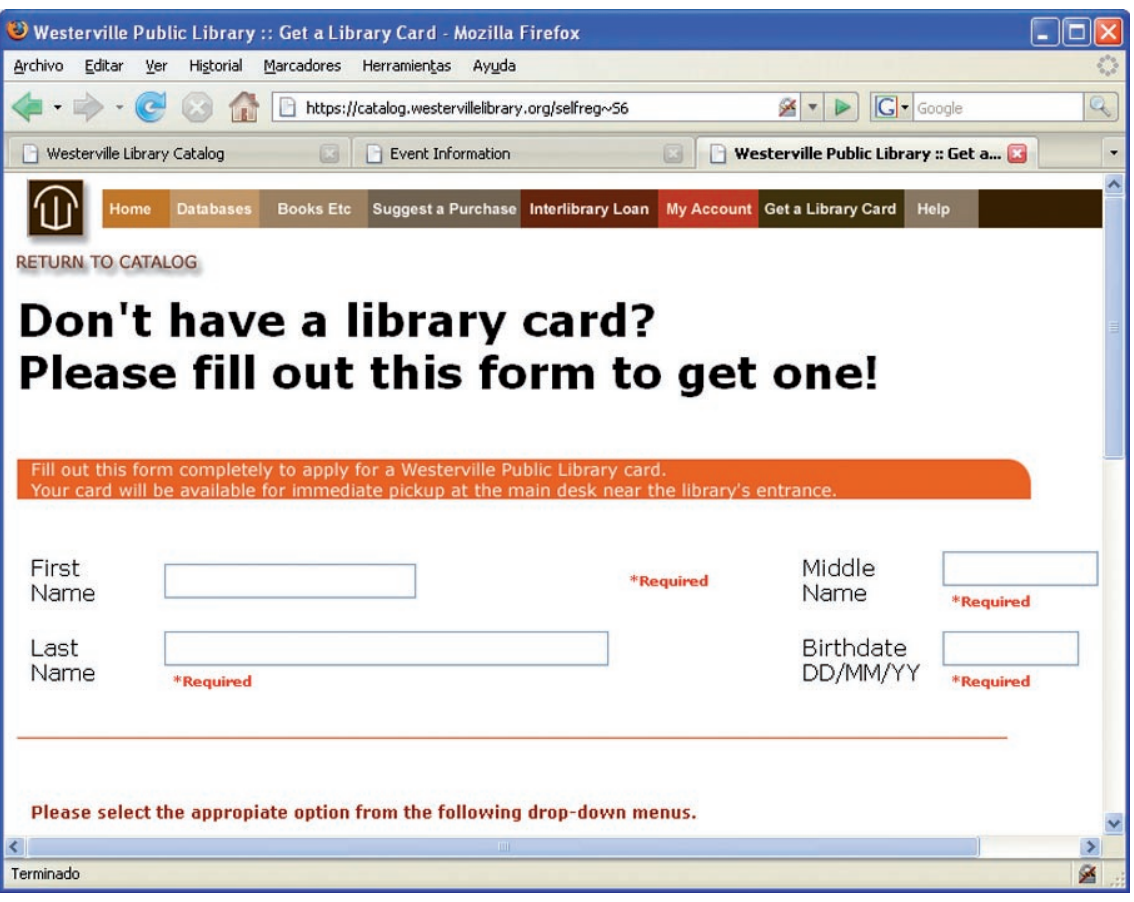

Formulario de auto-registro para usuarios de la biblioteca

terminales la información sobre sus reservas y préstamos actuales.

\section{“ExpressLane permite activar puestos de autoservicio para que sean los propios usuarios los que realicen sus préstamos"}

Es una solución interesante para las bibliotecas con un elevado número de préstamos así como una alternativa a las soluciones de autopréstamo de terceros, integradas a través del protocolo SIP2 ya que no requiere máquinas de autopréstamo. Los puestos donde se llevan a cabo están totalmente integrados con el sistema Millennium y conforme los usuarios completan las operaciones de préstamo y devolución, los datos se actualizan en la base de datos del catálogo en tiempo real.

Para ofrecer las máximas garantías de seguridad, ExpressLane puede configurarse para pedir a los usuarios un código PIN a la hora de tancia el papel que juega como un centro dinamizador de la actividad cultural de las comunidades, y es frecuente la organización de actividades de difusión cultural.

Program registration facilita el registro de información sobre los actos que se organizan -o que están relacionados con la biblioteca de una u otra forma-. Se pueden obtener datos sobre la localización, fechas, horarios, aforo, plazos para registrarse, precio -si lo tuviese- y un largo etcétera. Una vez dados de alta, los usuarios podrán recibir notificaciones y consultar un calendario a través del opac de Millennium, que permite el registro en línea de asistentes.

\section{"Program registration facilita a la biblioteca el registro de información sobre los distintos actos que se organizan"}

\section{Program registration}

El papel de la biblioteca pública está cambiando; ya no es únicamente un depósito de libros que los usuarios pueden tomar en préstamo. Cada vez tiene una mayor impor-
Incorpora funciones adicionales como la gestión de listas de espera -para aquellos acontecimientos que

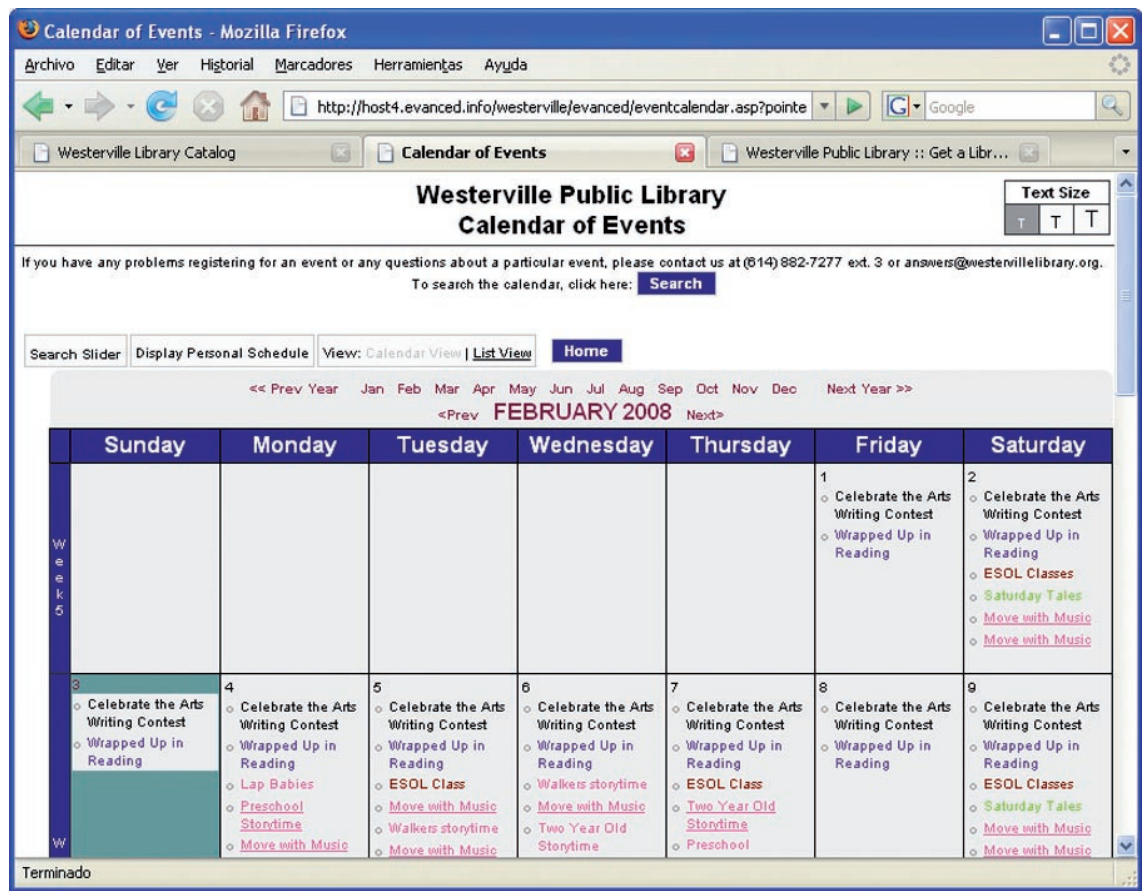

Calendario de eventos publicado por una biblioteca pública a través de Millennium 
hayan completado su aforo- y el envío de recordatorios a los usuarios registrados.

\section{Community manager patron review y Patron ratings}

Con este módulo, el opac deja de ser únicamente una herramienta para consultar la disponibilidad de ejemplares, al permitir una mayor interacción e intercambio de ideas entre los usuarios.

De esta forma será posible registrar reseñas de los libros en línea mediante unos formularios web que pasarán a estar disponibles para el resto de usuarios del sistema. Pueden establecerse controles y filtros para la revisión de las reseñas facilitadas por los usuarios y aprobarlas o rechazarlas (únicamente se mostrarán en el opac público aquellas que hayan sido autorizadas). Esta función está disponible únicamente para usuarios registrados, lo que impide la creación de reseñas anónimas. Cuando hay varias disponibles para un mismo libro se presentan en orden cronológico.

\section{"La biblioteca puede establecer controles y} filtros para la revisión de las reseñas facilitadas por los usuarios registrados, y aprobarlas o rechazarlas para que se muestren o no en el opac"

Esta funcionalidad completa la capacidad de los usuarios de crear evaluaciones, de 1 a 5 estrellas (rating) sobre los títulos de la colección. Cuando se ha evaluado un título, Millennium recalcula la media de estrellas atribuida por todos los usuarios y muestra la evaluación resultante en tiempo real. Los usuarios pueden consultar sus propias reseñas y sus evaluaciones en la interfaz personalizada: "Mi Millennium".

\section{Collection rotation para consorcios y redes}

Facilita la gestión de préstamos en consorcios o redes de bibliotecas públicas y, concretamente, el movimiento de las colecciones entre las bibliotecas de la red. Permite gestionar situaciones habituales en estos escenarios, como por ejemplo que los ejemplares sean prestados a una nueva ubicación (o biblioteca), y que posteriormente, mientras se mantiene activo ese préstamo, sean prestados de nuevo a otros usuarios. De esta forma un conjunto de ejemplares puede prestarse a una ubicación temporal donde podrán nuevamente ser prestados hasta que sean devueltos a su localización inicial.

Otra situación soportada por este módulo es agrupar varios ejemplares en una colección y determinar una secuencia de ubicaciones - cada una de ellas con una duración determinada- que se corresponden con la circulación prevista para esa colección. A partir de esta información se podrán gestionar las transferencias de ejemplares entre las ubicaciones previstas, y obtener listas para comprobar su situación regularmente.

Finalmente se puede realizar la gestión de colecciones "flotantes" permitiendo que determinados ejemplares permanezcan en la biblioteca donde se devuelve hasta su préstamo en otra diferente, independientemente de la de origen.

La gestión de las reservas es otra área de gran relevancia para los consorcios y redes de bibliotecas públicas. Innovative propone la funcionalidad de "Reservas regionales" (Regional holds). Cuando se crea una reserva sobre un título con múltiples ejemplares disponibles, se aplica sobre sólo uno de ellos. Se da preferencia al que tiene la prioridad más alta en la "región" del usuario y cuando en ésta no hay ejemplares disponibles, el sistema aplica la reserva al ejemplar disponible con prioridad más alta en cualquier otra. Esta función esta diseñada para minimizar el tiempo y la distancia recorrida para satisfacer una reserva. Finalmente, la función de "Círculos de reservas" (Request circles) permite aplicar el concepto de proximidad en la recogida de los ejemplares reservados. Se pueden definir grupos de bibliotecas y designar otras cercanas como centros de recogida propuestos a los usuarios, ofreciendo así un control sobre los puntos de entrega y recogida de ejemplares reservados.

\section{Interfaz para bibliotecas infantiles: KidsOnline}

Las bibliotecas infantiles necesitan adaptar sus interfaces de consulta para los más pequeños, y ofrecer pantallas fáciles de usar por los niños. La respuesta de Innovative a esta necesidad es KidsOnline. Una de sus singularidades es la función llamada Picture-it, que permite a los niños completar consultas usando imágenes en lugar de términos de búsqueda. Se trata de una interesante aproximación para facilitar la búsqueda de libros de forma intuitiva y gráfica, y ayudar a los niños a localizar obras que pueden resultar de su interés. La biblioteca puede modificar fácilmente la presentación visual de la interfaz sin necesidad de conocer lenguajes como html.

\section{Feed RSS}

Esta tecnología se ha generalizado en los últimos años para integrar en páginas web información procedente de otros sitios web. Un feed RSS es un documento xml que suele contener titulares de noticias, junto con una breve descripción y un hiperenlace que nos conduce a su texto completo. Desde nuestras páginas web podemos hacer referencia a ellos para que sus titulares se muestren en nuestras páginas 
en el lugar donde decidamos. Normalmente, aquellos recursos que consumen un feed RSS refrescarán periódicamente su contenido, descargando actualizaciones de manera automática.

En bibliotecas, pueden usarse de distintas formas:

- para incorporar al sitio web o al opac de la biblioteca titulares de noticias procedentes de otros sitios web, y

- para publicar -en forma de feeds RSS- información sobre las actividades que desarrolla la biblioteca, incorporación de nuevos fondos, etc., para que puedan ser consumidos por sitios web de terceros.

Millennium permite hacer estos dos usos de dicha tecnología. Por una parte, se podrán incorporar al sitio web de la biblioteca datos $R S S$ de otros sitios seleccionados por el bibliotecario, y los datos de la biblioteca también se pueden exponer en este formato mediante una herramienta llamada Feed builder. Esto permite alcanzar a un mayor número de usuarios y dar una mayor visibilidad a sus actividades. Además la incorporación de contenidos externos puede hacer de su web un sitio más atractivo para los usuarios.

\section{Encore}

Esta aplicación es el resultado de un análisis de las necesidades de grupos de usuarios de bibliotecas públicas y universitarias, destinado a conocer la forma en que éstos realizaban sus búsquedas para mejorar las interfaces de recuperación.

Permite a los usuarios registrar comentarios y valoraciones sobre los recursos de información, e incorpora un sistema de tagging para que asignen categorías o descriptores a los contenidos, los cuales serán revisados, aceptados o rechazados.

Los resultados de una búsqueda también se pueden acotar o redefi-

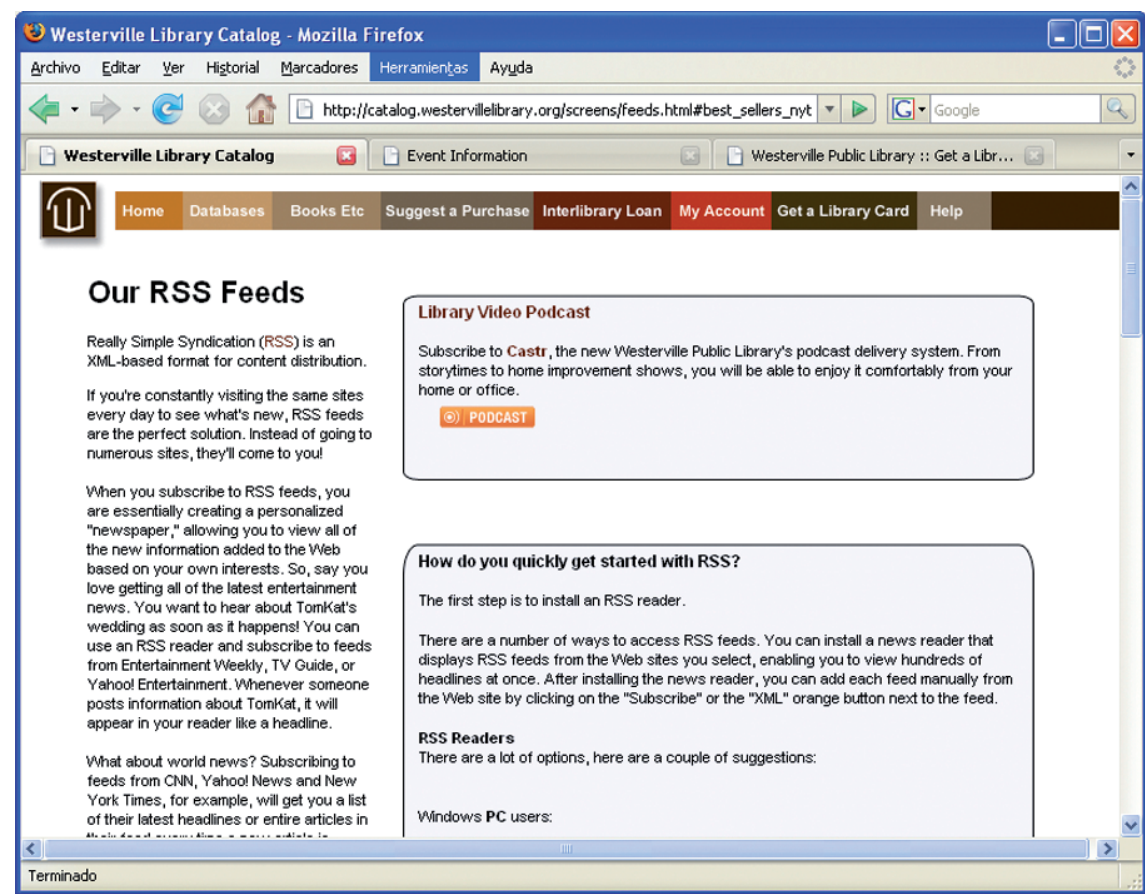

Feeds RSS incorporados al opac de la biblioteca

nir aplicando distintas facetas como el formato del recurso, colección a la que pertenece, idioma, o los descriptores que se les han asignado. La búsqueda incorpora una función similar al "¿Querías decir...?" habitual en los buscadores web, que hace comprobaciones ortográficas sobre los términos propuestos por el usuario y que presenta términos alternativos o complementarios a éstos.

\section{Conclusiones}

La empresa Innovative Interfaces Inc., ha desarrollado distintos módulos y aplicaciones informáticas que complementan a Millennium y que demuestran su compromiso con la innovación continua.

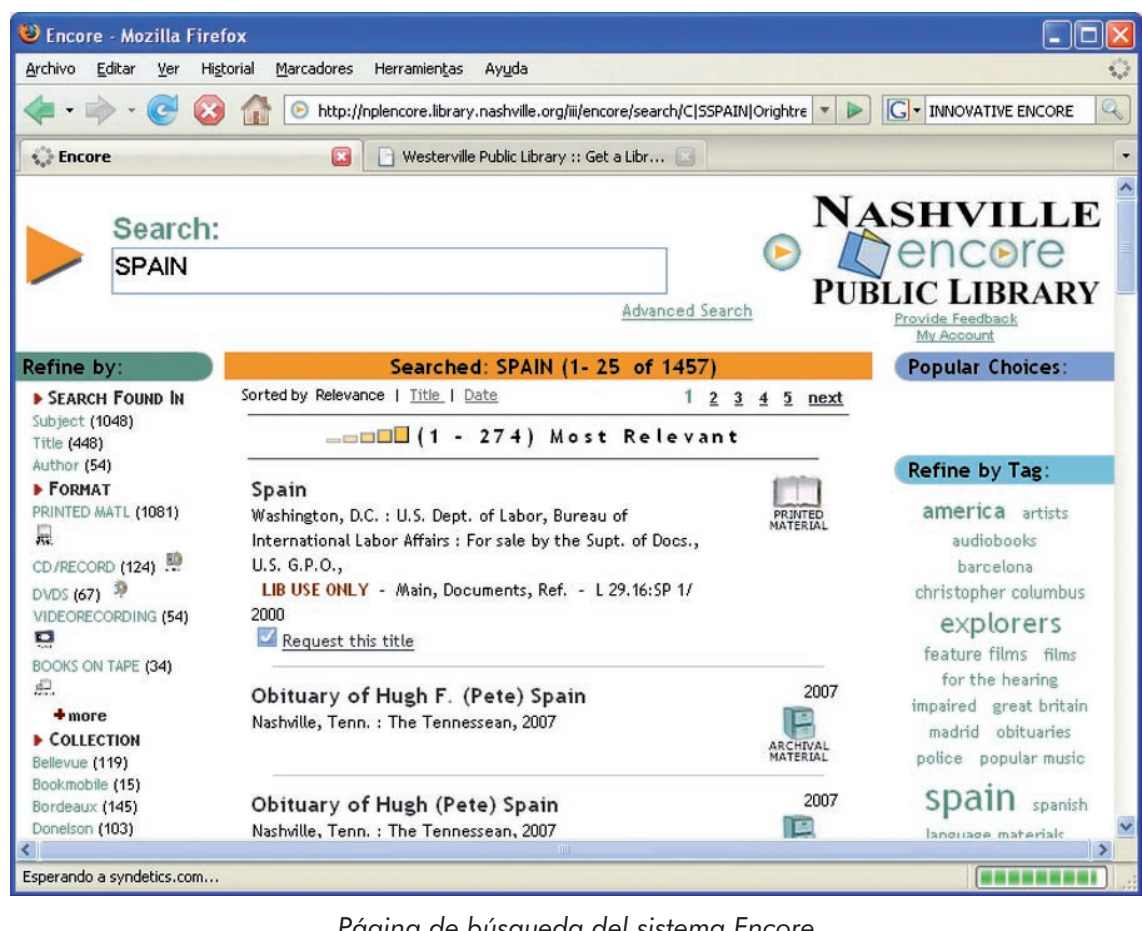

El profesional de la información, v.17, n. 2, marzo-abril 2008 


\section{Interfaz de consulta de Encore}

Se basa en una caja de consulta inicial donde el usuario puede teclear sus términos de búsqueda. El sistema devolverá una lista de resultados ordenados por relevancia y paginados. En la parte la izquierda se muestran una serie de facetas que permiten restringir o acotar los registros de la lista de resultados a aquellos que cumplan una serie de características: ser de un tipo determinado, pertenecer a una u otra colección, haber sido publicados en un año, escritos en un idioma particular, o aquellos donde los términos de búsqueda

figuran en los campos autor, título, etc.

En la parte derecha, se muestra la llamada tag cloud. Consiste en una serie de materias o categorías asignadas al conjunto de documentos recuperados (se presentan en un tamaño $\mathrm{u}$ otro dependiendo del número de documentos con los que están relacionados). El usuario puede hacer clic en sobre ellas para acotar los resultados de la búsqueda a aquellos registros a los que se hayan asignado dichas categorías.

En el área de las bibliotecas públicas, vemos cómo las principales novedades buscan facilitar la interacción con y entre sus usuarios (mediante la captura de reseñas, autotagging, o la organización de eventos), a la vez que se incorporan nuevas tecnologías de acceso a la red mediante dispositivos móviles. También ocupan un lugar desta- cado los sistemas de auto-servicio que buscan descargar a la biblioteca de actividades administrativas, rutinarias.

En resumen, se presenta una amplia oferta de soluciones técnicas que permiten situar a la biblioteca pública en el contexto que le exigen las últimas tendencias sociales y avances tecnológicos.
Para obtener más información de los productos de Innovative:

Maryvonne Enjolras, Plaza de la Basílica 19-9ª 28020 Madrid.

maryvonne@iii.com

Tel.: +34-915 559021

Ricardo Eíto-Brun, Universidad Carlos III de Madrid.

reito@bib.uc3m.es

\section{Potenciamos el valor de su información}

\section{Servicios} Automatización Tratamiento técnico Gestión integral Limpieza de fondos Outsourcing

\section{C/ Albadalejo, 40 Portal F} Madrid 28037

Tel.: 915062193 - ext 224 bibliodoc@bibliodoc.com

\section{Clientes}

\section{Archivos}

Bibliotecas

Centros de

Documentación

Empresas

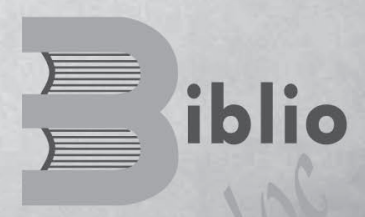

